\title{
Evaluación de Estudiantes para la Justicia Social. Propuesta de un Modelo
}

\author{
Student Assessment for Social Justice. Proposal of a Model
}

\author{
Avaliação de Alunos para a Justiça Social. Proposta de um \\ Modelo
}

\author{
Nina Hidalgo * \\ F. Javier Murillo
}

Universidad Autónoma de Madrid

\begin{abstract}
En este artículo se presenta una propuesta de Modelo de Evaluación de Estudiantes para la Justicia Social que busca avanzar un paso más en la construcción de una educación que contribuya a una transformación real y profunda de la sociedad. El modelo está basado en tres fuentes esenciales. Por un lado parte de un concepto de Justicia Social de carácter multidimensional, asentado en la Justicia social como Redistribución, como Reconocimiento y como Participación. La segunda fuente es una propuesta de Educación para la Justicia Social construida a partir del anterior concepto, que diferencia una educación en Justicia Social de una educación para la Justicia Social y que está conformada por tres ejes complementarios: una educación equitativa, crítica y democrática. Por último, la propuesta bebe y aprende de enfoques alternativos de evaluación de estudiantes: Evaluación Inclusiva, Auténtica, Culturalmente Sensible, Participativa, Democrático-Deliberativa y Crítica. Con todo ello se formula un modelo con tres dimensiones: evaluación equitativa, evaluación participativa y evaluación crítica. Esta propuesta pretende aportar ideas para empezar a transformar la evaluación de los estudiantes de tal forma que se configure como una pieza clave en una educación comprometida con la construcción de una sociedad más justa e inclusiva.
\end{abstract}

Palabras clave: Evaluación de estudiantes, Educación para la Justicia Social, Justicia Social, Evaluación equitativa, Evaluación crítica, Evaluación participativa.

This paper presents a proposal of a Model of Student' Assessment for Social Justice that seeks to go further in the construction of an education that contributes to a real and deep transformation of society. The model is based on three essential sources. On the one hand, it is part of a multidimensional concept of Social Justice, based on Social Justice as Redistribution, as Recognition and as Participation. The second source is a proposal of Education for Social Justice built from the previous concept, and differentiating an education in Social Justice from an education for Social Justice and which is made up of three complementary axes: an equitable, critical and democratic education. Finally, the proposal drinks and learns from alternative student assessment approaches: Inclusive, Authentic, Culturally Responsive, Participatory, DemocraticDeliberative and Critical Assessment. With this, a three-dimensional model is formulated: equitable assessment, participatory assessment and critical assessment. This proposal attempts to contribute in some ideas to begin to transform the students' assessment in such a way that it is configured as a key piece in an education committed with the construction of a fairer and more inclusive society.

Keywords: Student assessment, Social Justice education, Social Justice, Equitable assessment, Critical assessment, Participatory assessment.

*Contacto: nina.hidalgo@uam.es

ISSN: 2254-3139

www.rinace.net/riejs/

revistas.uam.es/riejs
Recibido: 5 de septiembre 2016

$1^{\text {a }}$ Evaluación: 22 de octubre 2016

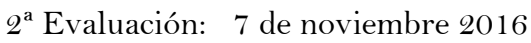

Aceptado: $\quad 10$ de noviembre 2016 
Este artigo apresenta uma proposta de um Modelo de Avaliação de Alunos para a Justiça Social, que busca dar mais um passo na construção de uma educação que contribua para uma real e profunda transformação da sociedade. O modelo é baseado em três fontes principais. Por um lado, parte de um conceito de justiça social multidimensional, sentado sobre a Justiça social como redistribuição, reconhecimento e, como participação. A segunda fonte é uma proposta de Educação para a Justiça Social construída a partir do conceito anterior, o que diferencia uma formação em educação na Justiça Social de uma educação para a Justiça Social e que consiste em três pilares complementares: uma educação equitativa, democrática e crítica. Finalmente, a proposta bebe e aprender com abordagens alternativas para a avaliação do aluno: avaliação Inclusiva, Autêntica, Culturalmente sensível, Participativa, Democráticadeliberativa e Critica. Com tudo isso formulamos o modelo tridimensional: Avaliação equitativa, a avaliação participativa e avaliação crítica. Esta proposta tem como objetivo trazer ideias para começar a transformar a avaliação dos alunos de uma maneira que a configure como uma parte fundamental de uma educação comprometida com a construção de uma sociedade mais justa e inclusiva.

Palavras-chave: Avaliação de Estudantes, Educação para a Justiça Social, Justiça Social, Avaliação equitativa, Avaliação crítica, Avaliação participativa.

\section{Introducción}

Es posible, es necesaria, otra evaluación de estudiantes: una evaluación democrática, no jerárquica, equitativa (que no igualitaria), positiva, formativa, del desarrollo integral... La evaluación define, y es definida por, los procesos de enseñanza y aprendizaje; forma parte indisolublemente de ellos. En la actualidad se han dado grandes pasos en la conformación de una Educación para la Justicia Social (p.ej., Adams, Bell y Griffin, 2007; Hackman, 2005; Zajda, Majhanovich y Rust, 2006) pero si no contamos con una evaluación con los mismos objetivos y características, todo esfuerzo por construir otra educación que nos lleve a otra sociedad será en vano.

Digámoslo de otro modo. La forma más eficaz de conseguir una educación que perpetúe y legitime las diferencias sociales es imponiendo evaluaciones externas, cuantitativas, centradas en materias instrumentales, iguales para todos... Los políticos lo saben, ¿cómo conseguir docentes sumisos y acríticos?, ¿cómo hacer que se enseñe todo y solo lo impuesto en un currículo cerrado no democrático?, ¿cómo acabar con la enseñanza creativa, crítica, trasformadora? Sencillo: con evaluaciones externas estandarizadas con repercusiones en la vida de los y las estudiantes y de los y las docentes.

La forma en que evaluemos a nuestros estudiantes, marcará inexorablemente su presente y su futuro:

Dime cómo evalúas y te diré qué sociedad construyes. La forma que tenemos de evaluar marca inexorablemente a nuestros estudiantes, en la escuela y a lo largo de toda su vida, y con ello se contribuye a crear una sociedad u otra. (Murillo e Hidalgo, 2015b, p. 5)

Es importante, no obstante, insistir en la idea de que la evaluación no puede entenderse de forma aislada, sino como un elemento más dentro de una Educación que verdaderamente trabaje para la Justicia Social. En este sentido, la evaluación forma parte de un proceso integrado en la enseñanza, por lo que no puede llevarse a cabo de forma separada a lo que se enseña, cómo se enseña y quién lo enseña. Por lo tanto, si y solo si la enseñanza tiene como propósito la consecución de una sociedad más justa es posible plantearse una evaluación con el mismo fin (Dochy, Segers y Dierick, 2002; Griffin, McGaw y Care, 2012; Sephard, 2000). 
En este artículo se presentan los fundamentos y el resultado del proceso de elaboración de un Modelo teórico de Evaluación de Estudiantes para la Justicia Social. En coherencia, el mismo se organiza en dos grandes apartados. Por una parte lugar se presentan las fuentes teóricas que sustentan el modelo y, por otra, se detalla el Modelo de evaluación de estudiantes para la Justicia Social propuesto.

\section{Fuentes fundamentantes del modelo}

Para la elaboración del Modelo de Evaluación de Estudiantes para la Justicia Social hemos realizado una revisión sistemática de la literatura relativa a tres fuentes principales: el concepto de Justicia Social, una propuesta de Educación para la Justicia Social y los enfoques alternativos de evaluación de estudiantes.

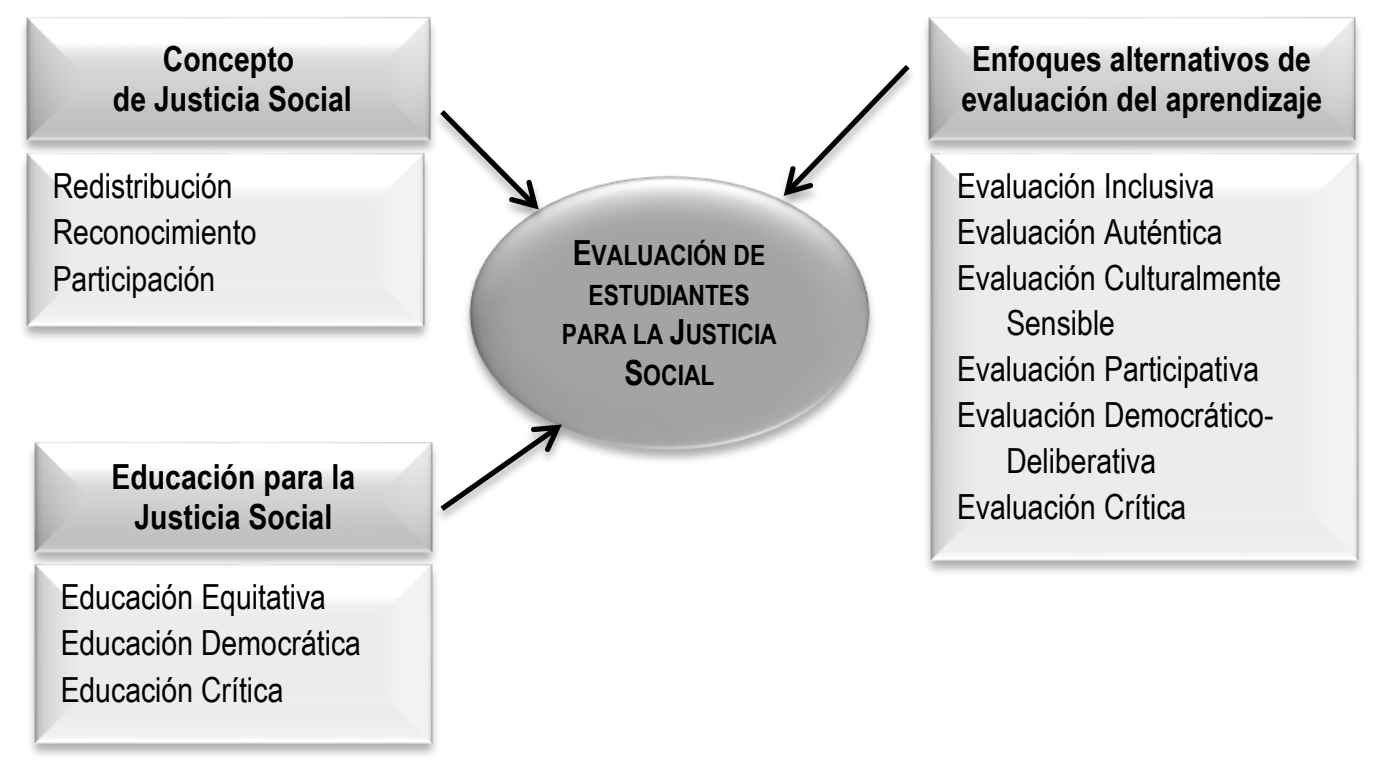

Figura 1. Fuentes del Modelo de Evaluación de Estudiantes para la Justicia Social Fuente: Elaboración propia.

\subsection{Primera fuente: concepto de justicia social}

Elaborar un Modelo de Evaluación de Estudiantes para la Justicia Social exige, en primer lugar, partir de un concepto de Justicia Social que oriente los elementos que lo conforman. Y ahí tenemos la primera dificultad: no existe una definición consensuada de lo que este término significa. Para comenzar podemos afirmar que la justicia se percibe cuando está ausente. Todos reconocemos la injusticia de un desahucio, de la pobreza infantil, de la violencia de género, de la homofobia... Pero esta percepción de las injusticias nos resulta insuficiente para avanzar. Un paso más es la confirmación de que Justicia Social es un concepto históricamente marcado, profundamente contextual y, sobre todo, claramente político; político en sentido freiriano, como una concepción envolvente del mundo y del ser humano).

Esta modelo parte del concepto de Justicia Social que el grupo de investigación "Cambio Educativo para la Justicia Social" viene trabajando en estos últimos años (Murillo y Hernández-Castilla, 2011). Así, basándonos en los planteamientos de la filósofa norteamericana Nancy Fraser (2008), entendemos la Justicia Social desde una 
perspectiva multidimensional conformada por tres elementos clave: Redistribución, Reconocimiento y Participación. Tres dimensiones mutuamente interdependientes, que se entrelazan y complementan aportándonos una noción de la Justicia Social multidimensional y compleja:

- Justicia Social como Redistribución, o Justicia Económica. Basada en los planteamientos canónicos de Rawls (1971, 2001) que entiende Justicia Social como distribución -o redistribución- equitativa de bienes primarios. Un concepto que se nutre de los aportes de Aristóteles y sigue con Ulpiano y Tomás de Aquino, pasando por Hegel y Marx, en la idea de "a cada uno según sus necesidades". Es decir, con una meta igualitaria mediante un proceso de desigualdad (dar más a quien por sus condiciones o su situación de partida más lo necesita). Desde esta dimensión, asumimos que un comportamiento socialmente justo exige un tratamiento diferencial que compense las desigualdades.

- Justicia Social como Reconocimiento, o Justicia Cultural. Se entiende como la ausencia de dominación cultural, del no reconocimiento o irrespeto de cualquier persona. Una justicia fundamentada en la valoración social y cultural de todos los individuos, así como de los diferentes modos de ser, hacer y pensar (Benhabib, 2006; Fraser y Honneth, 2003; Honneth, 1997, 2007; Taylor, 2003; Young, 2000).

- Justicia Social entendida como Participación, o Justicia Política. Se define como la plena participación de todos y todas en la vida social, en la toma de decisiones que les afectan. Esta participación es especialmente relevante para aquellos que han sido sistemáticamente excluidos sobre la base de su etnia, edad, género, habilidad física o mental, educación, orientación sexual, situación socioeconómica u otras características del grupo de pertenencia. Esta tercera dimensión es la que da sentido y vertebra las otras dos (Fraser, 2008; Young, 2011).

Como complemento, nos parece especialmente acertada la propuesta de la profesora Iris Marion Young (2000) que define la Justicia Social como la ausencia de opresión en la estructura social y en las instituciones. Y concreta este concepto de opresión en cinco dimensiones (las cinco caras de la opresión): explotación, marginación, carencia de poder, imperialismo cultural y violencia.

\subsection{Segunda fuente: la educación para la justicia social}

La segunda fuente que fundamenta el modelo es el enfoque de Educación para la Justicia Social que se defiende. De entrada una Educación para la Justicia Social ha de considerar una doble aproximación, que sea una Educación EN Justicia Social y DESDE la Justicia Social.

Efectivamente, de un lado, ha de ser una educación integral que forme a niños, niñas adolescentes y personas adultas EN Justicia Social, de tal forma que sea a través de ellos y ellas que se consiga esa sociedad más justa e inclusiva. Formar a personas que sean agentes de cambio, conocedores de las injusticias y sensibles ante ellas y con las competencias y motivación para cambiarlas (Adams, Bell y Griffin, 2007; Ayers, Quinn y Stovall, 2009; Hackman, 2005; Schniedewind y Davidson, 2006; Zajda, Majhanovich y Rust, 2006). Y, por otro, una educación cuya organización y funcionamiento (tanto en el 
sistema educativo como en los centros docentes o aulas) se base en los principios de redistribución, reconocimiento y participación antes comentados, una Educación DESDE la Justicia Social (Lupton, 2005; Murillo y Hernández-Castilla, 2014; North, 2006; Smyth, 2012).

Suscribiendo las palabras de Murillo y Hernández-Castilla (2014):

\begin{abstract}
La idea es sencilla, para construir una sociedad más justa es necesario que los alumnos conozcan esos elementos y tengan competencias y capacidades para modificar la situación, pero también mediante una escuela justa. Para entender esa idea podemos recurrir a la analogía de la educación para la democracia, si queremos una educación que contribuya a una sociedad más democrática necesitamos, en primer lugar, que los estudiantes conozcan cauces institucionales de participación, pero también que sepan expresarse en público para defender las ideas propias, así como actitudes favorables a la participación... entre otras cosas. Pero estaremos de acuerdo que no puede enseñarse democracia con instituciones autoritarias, por lo que es condición sine qua non que las escuelas sean democrática, que funcionen desde la democracia. (p. 18)
\end{abstract}

Como conjunción de las tres dimensiones que componen la Justicia Social y la doble perspectiva de una educación desde y en Justicia Social surge la necesidad de explicitar los componentes que conforman una educación para la Justicia Social. Estos tres componentes se concretan en una educación equitativa, una educación crítica y una educación democrática (figura 2 ):

- Educación equitativa, entendida como aquella que garantiza el acceso, la permanencia y el aprendizaje de todos los estudiantes, organizando y adaptando los recursos y medios de tal forma que ayude a aquellos alumnos que más lo necesitan (Bolívar, 2005; Murillo, 2004; Murillo y HernándezCastilla, 2014; Murillo y Román, 2008). Asimismo, la educación equitativa evita todas formas de exclusión, segregación, discriminación en un camino hacia una educación inclusiva, lo que supone una valoración y reconocimiento de las diferencias y fortalezas de los estudiantes (Ainscow, Dyson, Goldrick y West, 2013; Sleeter, 2013; Stowell, 2004).

- Educación democrática, concebida como aquella que es de todos y para todos y se fundamenta en la libertad social y la igualdad en la diversidad (Gutmann y Thompson, 2009; Knight y Pearl, 2000). Una educación democrática requiere procesos deliberativos y de toma de decisiones donde la participación y el trabajo cooperativo son esenciales (Apple y Beane, 1995; Belavi y Murillo, 2016; Biesta, 2015a; Friedrich, Jaastad y Popkewitz, 2010; Gutmann, 1987; Ruitenberg, 2009; Straume, 2015).

- Educación crítica, considerada como la que rompe con las relaciones de clase, las inequidades y la sumisión social y cultural existentes en la sociedad. La educación, desde una perspectiva crítica, tiene el poder de transformar y lograr la emancipación social de los estudiantes (Brisolera, 1998; Freire, 1978; Giroux, 1983, 1988; McLaren, 2003). Para lograr la emancipación social de los alumnos y alumnas la educación tiene que denunciar y transformar las situaciones de reproducción, legitimación, dominación y opresión generadas por cuestiones raciales, de clase, género u orientación sexual tanto en el sistema educativo como en la sociedad (Allman, 2001; Giroux, 1988; Duncan- 
Andrade y Morrell, 2008; Kincheloe, 2008; McLaren, 2003, 2015; McLaren y Kincheloe, 2007; Mezirow, 2000; Wink, 2005).

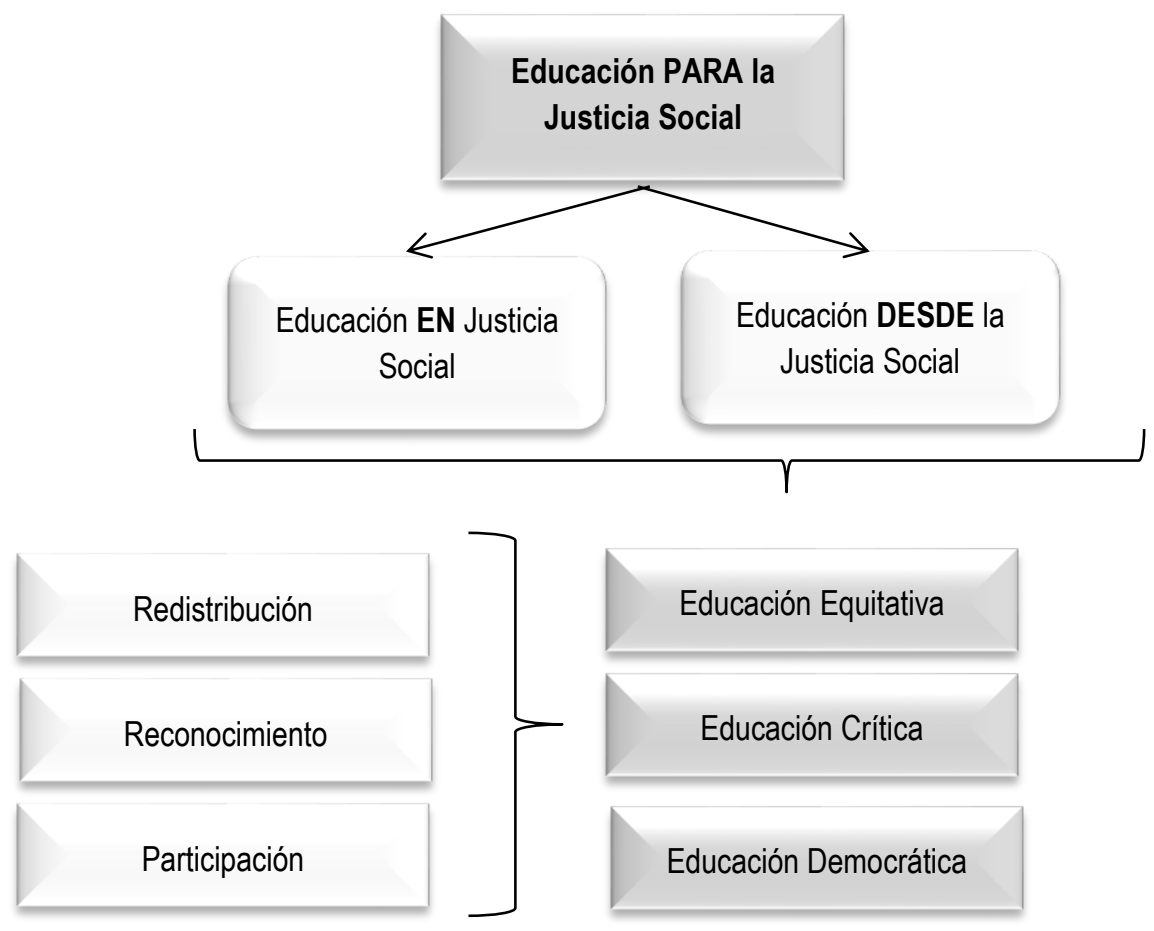

Figura 2. Elementos de una Educación para la Justicia Social Fuente: Elaboración propia.

\subsection{Tercera fuente: los enfoques alternativos de evaluación de estudiantes}

Como tercera fuente de inspiración, el modelo teórico se basa en diferentes corrientes de evaluación que se ha propuesto en estas últimas décadas en una necesidad de buscar otra forma de abordar este tema. Estos enfoques alternativos de evaluación se entienden como las perspectivas que han surgido en los últimos años que cuestionan la evaluación tradicional y proponen una evaluación que responda a las necesidades actuales de integrar e interpretar los aprendizajes por parte del estudiante y la transferencia de éstos a distintos contextos. Así, la evaluación desde un enfoque alternativo se caracteriza por ser útil, importante y significativa para los estudiantes. De estos enfoques de evaluación alternativa se han seleccionado los más influyentes para nuestro Modelo de Evaluación de Estudiantes para la Justicia Social:

a) Enfoque de evaluación inclusiva

Desde esta propuesta, la evaluación se convierte en un proceso individualizado donde se tiene en cuenta el punto inicial y final de los estudiantes, y se valora su progreso, pudiendo así adaptar la enseñanza a las necesidades de cada uno de ellos (Mertens, 1999, 2003; Ryan, Greene, Lincoln, Mathison y Mertens, 1999). La evaluación se concibe como un proceso integral y se adapta a que todos los estudiantes puedan desarrollarse al máximo. De este enfoque nos interesa que la evaluación se centre en el aprendizaje de los alumnos y alumnas, se adapte a los diferentes ritmos, necesidades y características de estos y que sirva para informarles de cómo se va desarrollando su aprendizaje (Goodwin, 
2012; Mertens, 1999, 2003; Murillo y Duk, 2012; Santiuste y Arranz, 2009; Saunders, 2006).

\section{b) Enfoque de evaluación auténtica}

La evaluación auténtica es aquella que plantea problemas interesantes y cuestiones de importancia para los estudiantes, en la que éstos deben utilizar el conocimiento para llevar a cabo acciones eficaces y creativas para resolverlos. Desde un enfoque de evaluación auténtica, las actividades son réplicas a los tipos de problemas que se enfrentan los alumnos y alumnas en la vida real (Darling-Hammond y Snyder, 2000; Janesick, 2008; Wiggins, 1993). Asimismo, la evaluación tiene que ser más holística y estar vinculada con los intereses de los estudiantes así como ser práctica y útil para su vida. De una evaluación auténtica nos centramos concretamente en el carácter práctico de la evaluación (siendo significativa para el estudiante) así como en evaluar al alumnado de forma integral (Aitken y Pungur, 2001; Gulikers, Bastiaens y Kirschner, 2004; Mueller, 2006; Swaffield, 2011; Janesick, 2006, 2008; King, Schroeder y Chawszczewsky, 2001; Torrance, 1995; Vu y Dall'Alba, 2014).

\section{c) Enfoque de evaluación culturalmente sensible}

Este enfoque plantea que la evaluación es una actividad altamente vinculada al contexto en el que se desarrolla y por ende tiene que centrarse en el entorno y en la realidad cultural y social de los alumnos y alumnas. Así, a través de la evaluación, se trabaja y valora el conocimiento cultural, las experiencias y la diversidad étnica de los estudiantes, favoreciendo la mejora de la autoestima de todos ellos (Frierson, Hood y Hugues, 2002; Gay, 2010; Kozleski, 2010; Thomas y Stevens, 2004). De este enfoque nos interesa especialmente el carácter cultural y social que asume la evaluación, valorando y respetando las diferentes identidades, costumbres y creencias de los estudiantes.

\section{d) Enfoque de evaluación participativa}

La evaluación participativa surge del campo de la investigación social, concretamente de la investigación participativa y la investigación-acción participativa. El enfoque participativo de la evaluación se basa en el empoderamiento de los estudiantes, es decir, la colaboración activa de estos en todas las fases del proceso evaluativo. Los alumnos y alumnas participan en la toma de decisiones de su evaluación favoreciendo su implicación y motivación en su aprendizaje (Burke, 1998; Cabrera y Bordas, 2001; Cousins y Whitmore, 1998; Greene, 2006; Jackson y Kassam, 1998; Pfeiffer, 2007; Sabirón, 1999; Whitmore, 1998). De este enfoque nos interesa especialmente la implicación de los estudiantes en todas las fases del proceso evaluativo, su participación activa que les empodere y les de voz en su aprendizaje y la perspectiva dialógica y de consenso que aporta este enfoque a la evaluación.

\section{e) Enfoque de evaluación democrático-deliberativa}

Este enfoque entiende la evaluación como un medio y un fin para contribuir al avance de la democracia en la sociedad (House y Howe, 2000a, 2000b). Desde la perspectiva de sus autores más representativos, House y Howe (2000a), la evaluación se concibe como una actividad altamente vinculada con la autoridad de las estructuras sociales y que fácilmente contribuye a mantener procesos autoritarios, jerárquicos e injustos. Frente a esta situación, la evaluación tiene el propósito de garantizar la implicación de los estudiantes en todo el proceso evaluativo, teniendo en cuenta sus opiniones, ideas y 
puntos de vista para llevar a cabo una toma de decisiones consensuada. Solo con la participación de los estudiantes, la evaluación tiene el poder de redistribuir el poder en el aula y denunciar la jerarquía social existente (House y Howe, 2000b). De este enfoque, aprendemos para nuestro modelo la importancia de que la evaluación sea un proceso altamente democrático, que la toma de decisiones sea consensuada e incluya los distintos puntos de vista y formas de pensar de los estudiantes y que el poder se distribuya entre los alumnos y alumnas, convirtiéndolos en protagonistas de su aprendizaje.

\section{f) Enfoque de evaluación crítica}

A partir de esta propuesta, la evaluación se concibe como un proceso altamente político y moral, por lo que tiene consecuencias no solo a nivel educativo sino también social. Desde la perspectiva crítica, la finalidad última de la evaluación es la emancipación social de los estudiantes (Giroux, 2003; McLaren, 2003). Las aportaciones al modelo de este enfoque se centran en el carácter político de la evaluación, la importancia de incluir en la evaluación elementos que susciten la denuncia social y la necesidad de promover una conciencia crítica en los estudiantes a través del proceso evaluativo para que reflexionen, denuncien y actúen en contra de las injusticias sociales (Everitt, 1996; Everitt y Hardiker, 1996; Freire, 1978, 1979; McLaren, 1997, 2003; Ryan, 2004; Waters, 1998).

La Evaluación de Estudiantes para la Justicia Social se inspira en los anteriores enfoques y los integra dando un paso más allá. No se trata únicamente de destacar los elementos más relevantes de cada uno de ellos, sino reinterpretarlos a la luz de nuestro concepto de Justicia Social dotándoles de nuevas potencialidades.

\section{Hacia un modelo de evaluación de estudiantes para la justicia social}

El modelo teórico de Evaluación de Estudiantes para la Justicia Social está conformado por tres dimensiones: evaluación equitativa, evaluación participativa y evaluación crítica.

\subsection{Evaluación equitativa}

Una evaluación socialmente justa pasa necesariamente por ser equitativa. Evaluar a todos los y las estudiantes de la misma forma dista mucho de la justicia. Una evaluación para la Justicia Social se adecúa a cada uno de ellos y ellas identificando las ayudas y recursos necesarios que requieren para facilitar su aprendizaje. Así, la evaluación no es igual para todas las personas, sino que supone dar más apoyo o recursos a aquellos estudiantes que por sus características sociales o situación de partida más lo necesitan. Esta adaptación o individualización de la evaluación se complementa con que esta sea sensible a sus características: su cultura, su género, su estilo de aprendizaje, orientación sexual... (Álvarez Méndez, 2001; Goodwin, 2012). Asimismo, para llevar a cabo una evaluación de los estudiantes para la Justicia Social es necesario que sea integral, atendiendo al desarrollo cognitivo y socio-afectivo del estudiante así como continua y cualitativa, favoreciendo una evaluación sistemática que ayude a los alumnos a mejorar su proceso aprendizaje

Concretando todos estos elementos mencionados, una evaluación equitativa desde un enfoque de Justicia Social se caracteriza por ser integral, adaptada, culturalmente sensible, cualitativa, continua y del progreso. 


\section{a) Integral}

Una evaluación socialmente justa concibe de forma integral al estudiante, considerando no tan solo los aspectos cognitivos (el saber), sino también las habilidades y procedimientos (el saber hacer), los aspectos afectivos (el saber ser), los valores sociales (las actitudes, los valores y las normas), los aspectos psicomotores, la creatividad, las expectativas, el interés, la motivación y el sentido crítico. Evaluar al estudiante de forma integral supone que los instrumentos y estrategias utilizados sean variados para que los estudiantes puedan demostrar lo que han aprendido desde diferentes perspectivas. Además, la evaluación tiene un carácter holístico ya que tiene en cuenta todos los elementos que inciden en ella: los objetivos, los procesos e estrategias utilizadas, los recursos y el contexto en el que se encuentra el estudiante. Una evaluación integral es también transversal, es decir, que evalúa diferentes áreas curriculares y tiene un carácter interdisciplinario y globalizador, favoreciendo el desarrollo de una mentalidad global, superando la visión fragmentada de la educación (Murillo e Hidalgo, 2015a; Sadler, 2009).

\section{b) Adaptada}

Otra característica de una evaluación socialmente justa es que esta se adapta a las necesidades y características de los alumnos y alumnas en todo el proceso evaluativo (Santiuste y Arranz, 2009). Durante el diseño de la evaluación se adecuan las pruebas de evaluación al estilo de aprendizaje, capacidades e intereses de los alumnos. En la ejecución del proceso evaluativo las condiciones y formas de aplicar la prueba pueden ser cambiantes, ofreciendo distintas posibilidades para que la evaluación pueda llevarse de tal forma que les ayude a todos. En la corrección de la evaluación, esta se adapta a su nivel, sus intereses y sus expectativas, pudiendo variar los criterios de corrección y calificación entre los estudiantes (Mertens, 2003; Saunders, 2006).

\section{c) Culturalmente sensible}

La evaluación para la Justicia Social, debe ser sensible con la cultura, género, capacidad, orientación sexual y clase social de los estudiantes. Una evaluación culturalmente sensible incluye elementos, características y contenidos que respeten a todos y cada uno de los estudiantes (Irvine y Armento, 2001). Supone, también, un reconocimiento explícito de los estudiantes en el aula, recogiendo, valorando y destacando sus características, peculiaridades y fortaleciendo su identidad. En este sentido, las actividades de evaluación son congruentes con sus antecedentes culturales, sociales e ideológicos (Frierson, Hood, y Hugues, 2002; Sleeter, 2013; Thomas, 2004; Thomas y Stevens, 2004).

\section{d) Cualitativa}

La evaluación adquiere un carácter descriptivo, narrativo e interpretativo y pone el énfasis en los aspectos subjetivos del aprendizaje de los estudiantes. La evaluación no busca la objetividad sino la credibilidad del aprendizaje de cada uno de alumnos y alumnas. Complementando esta idea, la evaluación cualitativa es naturalista, es decir, que evalúa al estudiante en su contexto natural y cómo este incide en su aprendizaje, valorando no tan solo el contenido aprendido sino también la forma en la que cada alumno lo adquiere y lo aplica académica y contextualmente, es decir, involucrando la significación de los aprendizajes (Cabrera y Bordas, 2001). Otro aspecto fundamental es que se centre más en el proceso que en el resultado de aprendizaje. Por último, la devolución de la 
información en la evaluación cualitativa es descriptiva, ofreciendo una rica descripción del proceso de aprendizaje (Moreno Olivos, 2004, 2010).

\section{e) Continua}

La evaluación es una actividad constante que se desarrolla a lo largo de toda la enseñanza y no de forma puntual al final de una unidad didáctica o un trimestre. El proceso evaluativo se caracteriza por su carácter formativo, es decir, que su objetivo es perfeccionar el proceso de formación de los estudiantes. Este carácter formativo supone una toma de decisiones constante que favorece que los estudiantes sean conscientes del avance y las dificultades de su proceso de aprendizaje y así poder cambiar, mejorar o enriquecer su propio proceso (Black y William, 2009). La evaluación continua conlleva una reflexión constante entre docentes y estudiantes para mejorar de forma conjunta la enseñanza y por ende el aprendizaje. Asimismo, supone una retroalimentación constante del proceso, ayudando al estudiante a regular su aprendizaje (Bell y Cowie, 2001; Black y William, 2009; Sephard, 2005).

\section{f) Del progreso}

Muy ligado con la anterior característica, una evaluación socialmente justa valora el progreso de los estudiantes en su aprendizaje por encima de los resultados (Murillo e Hidalgo, 2015b). Una evaluación del progreso de los estudiantes permite conocer la línea de aprendizaje de cada estudiante, es decir, el punto de partida de cada uno de ellos, qué camino han ido siguiendo, cuáles han sido sus puntos fuertes y débiles, cómo los ha afrontado y qué resultado han obtenido. Evaluar el recorrido de aprendizaje de cada estudiante supone tener en cuenta el esfuerzo, la implicación y la motivación que cada uno de ellos ha mostrado en el aprendizaje y evaluarlo más allá de los resultados obtenidos (Cabrera y Bordas, 2001; Murillo e Hidalgo, 2015b; William, 2007).

En ese sentido es muy importante que sea útil y práctica, que de verdad contribuya a que estudiantes y docentes tengan información para optimizar el aprendizaje (Janesick, 2008).

\subsection{Evaluación participativa}

Supone avanzar de una evaluación de los estudiantes a una evaluación con ellos. Una evaluación para la Justicia Social exige que los estudiantes participen y decidan en todas las fases de la evaluación (desde el diseño hasta la devolución de la información generada por el proceso evaluativo); se trata de generar su empoderamiento que les haga ser actores y autores de su proceso de aprendizaje y evaluación. Este dar voz a los estudiantes significa que los docentes incluyan sus perspectivas, tomen decisiones con ellos de forma consensuada y busquen soluciones de forma conjunta para mejorar tanto el aprendizaje individual como grupal. Una evaluación participativa convierte a los estudiantes en protagonistas de su aprendizaje, participando activa y autónomamente en su evaluación. Para ello es necesario, por un lado, crear un entorno de confianza y positividad donde los alumnos se sientan cómodos para expresar tanto sus ideas como sus opiniones y por otro, llevar a cabo una evaluación cooperativa donde los estudiantes trabajen conjuntamente con los docentes para mejorar su aprendizaje.

Desde esta perspectiva la Evaluación Participativa se caracteriza por ser democrática, dialógica y cooperativa. 


\section{a) Democrática}

El proceso de evaluación es compartido por estudiantes y docentes con el objetivo de buscar una evaluación que beneficie a todos. De este modo, los estudiantes se convierten en sujetos activos de su propio aprendizaje (Cabrera y Bordas, 2001; King, Cousins y Whitmore, 2007; Sabirón, 1999). La evaluación democrática supone la implicación de los estudiantes en todas las fases del proceso evaluativo: el diseño, la ejecución, la corrección y la devolución de la información de la evaluación. Para que los alumnos y alumnas se impliquen en su aprendizaje hay que cederles responsabilidad, lo que supone que la toma de decisiones esté en manos de los docentes y de los estudiantes. Las decisiones que se toman en la evaluación democrática son de forma consensuada, es decir, a través de un acuerdo alcanzado por consentimiento entre los alumnos y los docentes en temas relativos al momento, instrumentos y criterios de evaluación, tratando siempre de minimizar el conflicto que pueda surgir por las opiniones sustentadas por la minoría (Pfeiffer, 2007). Uno de los principales beneficios del consenso es que estimula el debate democrático, favoreciendo la existencia de situaciones y momentos donde los estudiantes ofrecen su punto de vista de forma razonada y argumentada (Cabrera y Bordas, 2001; Gutmann y Thompson, 2009; Whitmore, 1998).

\section{b) Dialógica}

Otra característica de una Evaluación de Estudiantes para la Justicia Social es que esta utilice el diálogo como elemento clave en la participación de los estudiantes en el proceso evaluativo (Sabirón, 1999). El diálogo se concibe como la herramienta principal para que los estudiantes y el docente expresen de forma libre las opiniones y puntos de vista para llegar a un acuerdo común. Se trata de un planteamiento evaluativo interactivo y comunicativo basado en la igualdad y que persigue la participación de alumnos y docentes en la toma de decisiones. Para llevar a cabo una evaluación dialógica es necesario crear un clima de confianza, libertad y flexibilidad, donde cada alumno valore su aportación y respete las de sus compañeros y docentes (Hodgen, 2007). Crear un entorno positivo, cercano y seguro favorece que los estudiantes se sientan cómodos para expresar sus ideas y opiniones y de esta forma se impliquen en el proceso evaluativo. Cobra especial relevancia que la retroalimentación de la evaluación se lleve a cabo también de forma dialógica, traduciéndose en un intercambio interactivo en el que se comparten las interpretaciones, significados negociados y las expectativas relativas a los aprendizajes adquiridos por los estudiantes. La evaluación puede y debe convertirse en una plataforma de debate sobre la enseñanza, donde intervengan las familias, los estudiantes y los docentes (Cabrera y Bordas, 2001; Hodgen, 2007; Stern y Backhouse, 2011).

\section{c) Cooperativa}

La evaluación participativa se define por el uso de estrategias de evaluación cooperativas, que supongan el trabajo conjunto de los estudiantes y el desarrollo de habilidades de trabajo en equipo, resolución de conflictos etc. Las técnicas cooperativas de evaluación que se pueden desarrollar son diversas, desde exámenes colaborativos hasta trabajos grupales pasando por la resolución de problemas (Griffin, McGaw y Care, 2012; Hargreaves, 2007; Swan, Shen y Hiltz, 2006). Asimismo, la evaluación cooperativa también supone la cooperación y colaboración entre docentes y estudiantes. La evaluación socialmente justa es un proceso de construcción compartida que facilita tanto la consecución de los objetivos consensuados como del aprendizaje de cada uno de ellos. 
Una evaluación cooperativa fomenta el uso tanto de la autoevaluación y como de la coevaluación. Por un lado, la autoevaluación es una estrategia que les ayuda a valorar, criticar y a reflexionar sobre el proceso de enseñanza y aprendizaje individual mediante la interacción entre docente y estudiantes. Por otro lado, la coevaluación o evaluación entre iguales es un proceso de valoración conjunta que realizan los estudiantes sobre la actuación del grupo, atendiendo a criterios de evaluación o indicadores establecidos por consenso. La coevaluación permite que los alumnos ponderen el desempeño de sus compañeros, favoreciendo el desarrollo de una mentalidad crítica, reflexiva y responsable tanto de su propio aprendizaje como del de sus compañeros (Dochy, Segers y Sluijmans, 1999; Leach, Neutze y Zepke, 2001; Lejk y Wyvill, 2001; Nicol y Macfarlane-Dick, 2006).

\subsection{Evaluación crítica}

Una evaluación que luche para eliminar las injusticias en la sociedad es imprescindible que sea crítica. La evaluación es una actividad ligada directamente con el poder social y político que legitima las diferencias existentes en la sociedad. Así, cuando la evaluación es crítica puede promover que los estudiantes sean conscientes de las desigualdades y problemas que les rodean. Para ello, es necesario repensar los contenidos que se trabajan y evalúan en el aula con el objetivo de que los estudiantes sean críticos con la realidad y la transformen. Una evaluación crítica se caracteriza por ser política, no jerárquica, transformadora y emancipadora.

\section{a) Política}

La evaluación de los estudiantes no es una actividad aislada, sino que tiene relaciones directas con la política, la economía y la sociedad. Los centros educativos se conciben como espacios profundamente morales y políticos donde los docentes muestran sus visiones específicas de la vida pública, la comunidad y la responsabilidad moral (Freire, 1972; House, 1997). La forma en que los profesores entienden la política y la sociedad se refleja claramente en cómo conciben la evaluación. Así, la evaluación refleja los intereses políticos existentes en la sociedad. La evaluación es un mecanismo que transmite los valores dominantes y organiza a los estudiantes y la sociedad en base a dichos valores. Las decisiones que los docentes toman en el aula repercuten directamente en la vida del estudiante, ordenándolo y situándolo en una posición de poseedor o no del conocimiento considerado como válido. Desde una perspectiva de Justicia Social, la evaluación ayuda a que los estudiantes sean conscientes y críticos con el carácter político de la evaluación y cómo influye en sus vidas (Apple, 1986; Freire, 1972, 1978; House, 1997; Santos Guerra, 1993, 2003).

\section{b) No jerárquica}

Otro elemento muy vinculado al carácter político de la evaluación es que evidencia las relaciones de poder que se dan en el proceso evaluativo. La jerarquía que se establece a través de la evaluación genera una situación de desigualdad entre docente y estudiantes, donde el profesor tiene el poder y los estudiantes asumen de forma pasiva dicha evaluación. Una evaluación socialmente justa cuestiona las relaciones de poder que genera la evaluación y sitúa a docentes y estudiantes en el mismo nivel para que las decisiones que se tomen sean de forma conjunta, eliminando las jerarquías impuestas por el sistema social (House, 1997). 


\section{c) Transformadora}

Una evaluación para la Justicia Social promueve que estudiantes y docentes reflexionen de forma conjunta sobre proceso evaluativo que se lleva a cabo en el aula. Es necesario que la reflexión se refiera a todas las fases de la evaluación: el diseño, los criterios de evaluación, los instrumentos y estrategias que se van a emplear para valorar el conocimiento adquirido por parte de los alumnos, la ejecución de la evaluación, la corrección y la devolución de los resultados (Torrance, 2012). La evaluación es, por lo tanto un proceso reflexivo y crítico compartido que concluye en la transformación de la propia evaluación, de la enseñanza de los docentes y del aprendizaje de los estudiantes (Popham, 2011; Wehlburg, 2008).

\section{d) Emancipadora}

Uno de los propósitos fundamentales de la evaluación socialmente justa es que genere una conciencia crítica en los alumnos y alumnas. Despertar esta conciencia supone que los estudiantes no tan solo conozcan las injusticias sino que también sean capaces de analizarlas, ser críticos con ellas y comunicarlas a la sociedad (Giroux, 1988). Solo cuando los estudiantes tienen una conciencia crítica con las injusticias existentes en la sociedad son capaces de llevar a cabo acciones que cambien la sociedad, es decir, que se conviertan en agentes activos de cambio. La evaluación emancipadora, por lo tanto, supone que los estudiantes sean críticos con la realidad que les rodea y actúen para transformarla.

Una imagen global y comprensiva del modelo propuesto de Evaluación de Estudiantes para la Justicia Social se muestra en la figura 3.

\section{A modo de cierre}

En este artículo hemos formulado una propuesta de un Modelo de Evaluación de Estudiantes para la Justicia Social que pretende convertirse en un paso más en la construcción de una educación y una enseñanza alternativas que contribuyan a una sociedad más justa e inclusiva. Dicho modelo se fundamente en tres elementos interrelacionados: un concepto multidimensional de Justicia Social, una propuesta de Educación para la Justicia Social basada en el anterior concepto y las aportaciones de los enfoques alternativos de evaluación de estudiantes. De cada uno de ellos se han obtenidos ideas y componentes, en el intento de aportar un modelo globalizador, pero que no se queda en la sume de sus partes, sino en una nueva aportación.

Con ello imaginamos una evaluación, en primer lugar, equitativa; es decir, una evaluación del desarrollo integral, adaptada, culturalmente sensible, cualitativa, continua y que valore el progreso del estudiante, no el punto de llegada. Pero también que sea una evaluación participativa: democrática, dialógica y cooperativa. Y, por último, que sea crítica, que contribuya a cambiar, explícitamente política, no jerárquica, transformadora y emancipadora. Es, por todo ello, una utopía necesaria. Un camino hacia el que andar para hacer otra evaluación y otra educación.

Cierto es que, en ocasiones se ha relacionado el enfoque de Evaluación Auténtica como la mejor aproximación evaluativa para una educación para la Justicia Social (p.ej., DarlingHammond, 1994; Lingard y Mills, 2007; Lingard, Mills y Hayes, 2000; Rennert-Ariev, 2005). Sin embargo, desde nuestra perspectiva esta aproximación es insuficiente por no 
contar con elementos claves en una enseñanza de verdad transformadora. Hacemos referencia, por ejemplo a una evaluación participativa y democrática; o a un enfoque crítico de la evaluación, que le dota a la misma de una potencialidad que, desde nuestro punto de vista está ausente del enfoque de Evaluación Auténtica. Algo parecido puede decirse de enfoques como el de Evaluación Democrática (Biesta, 2015b) o el de Evaluación Crítica, los cuales a su vez carecen de elementos como la evaluación auténtica o inclusiva.

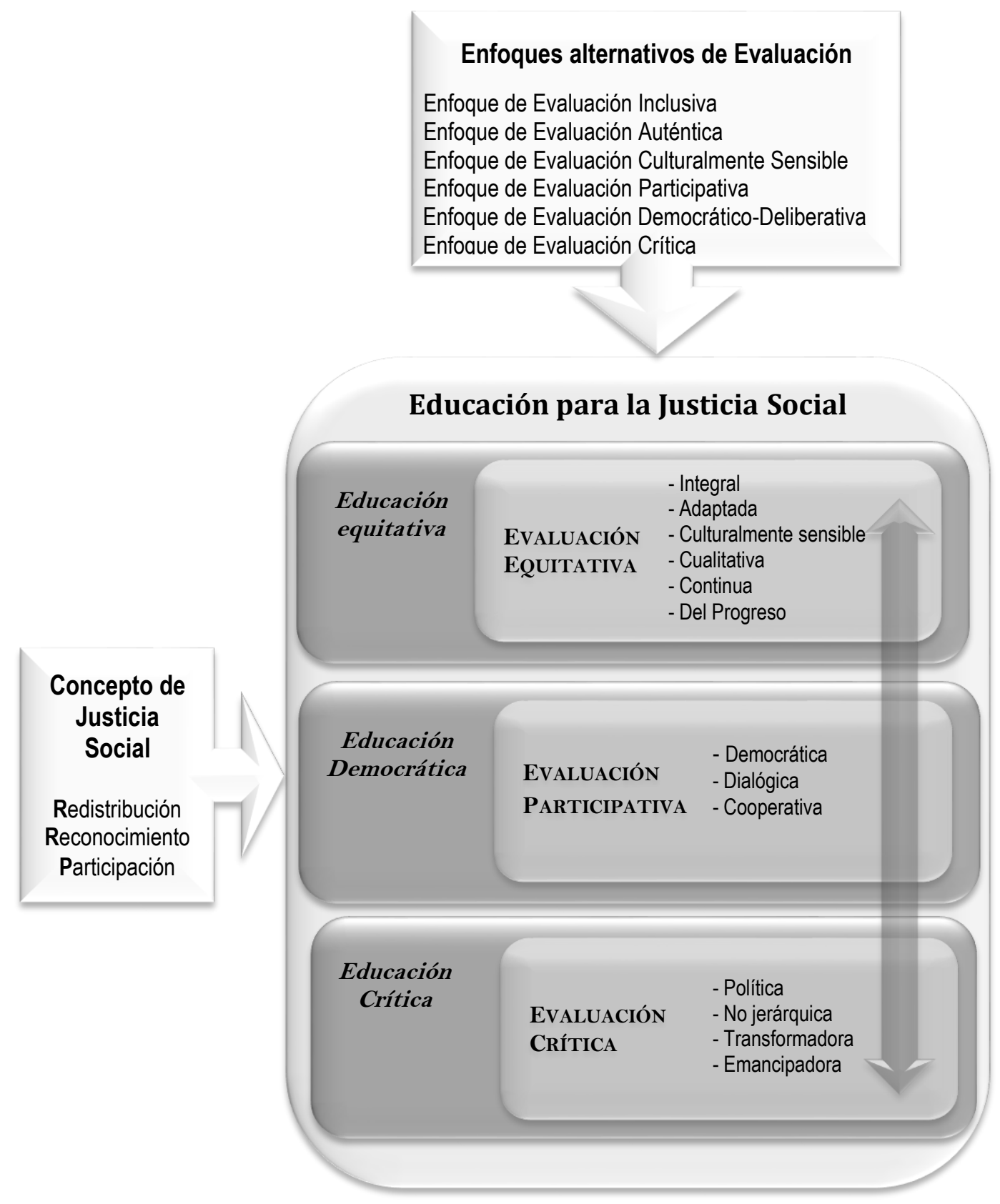

Figura 3. Modelo de Evaluación de Estudiantes para la Justicia Social Fuente: Elaboración propia.

La propuesta formulada a través de este artículo es solo eso, una propuesta que se queda en el desarrollo teórico. La misma obtendrá todo su potencial a partir de dos desarrollos. De un lado, a partir de la validación empírica de la misma. Una validación por parte de 
docentes y especialistas en la temática se constituye como el siguiente peso necesario. También es necesario hacer un esfuerzo por concretar esta propuesta en estrategias, instrumentos, propuestas detalladas que sirven de forma real a ayudar a los y las docentes a hacer una evaluación de los estudiantes diferente.

La evaluación marca la dinámica del aula, ya que define la forma de enseñar y los comportamientos y actitudes de los y las estudiantes. No hay alternativa, si queremos una educación que de verdad contribuya a crear una sociedad más justa, necesitamos una evaluación que trabaje en esa misma dirección.

\section{Referencias}

Adams M., Bell, L. A. y Griffin P. (2007). Teaching for Diversity and Social Justice. Nueva York, NY: Routledge.

Ainscow, M., Dyson, A., Goldrick, S. y West, M. (2013). Developing equitable education systems. Nueva York: Routledge.

Aitken, N. y Pungur, L. (2001). Authentic Assessment. Recuperado de http://education.alberta.ca/apps/aisi/literature/pdfs/AuthenticAssessmentUofAbUofL.P $\mathrm{DF}$

Allman, P. (2001). Critical education against global capitalism: Karl Marx and revolutionary critical education. Londres: Bergin and Garvey.

Álvarez Méndez, J. M. (2001). Evaluar para conocer, examinar para excluir. Madrid: Morata.

Apple, M. W. (1986). Ideología y currículo. Madrid: Ediciones Akal.

Apple, M. W. y Beane, J. A. (1995). Democratic schools. Alexandria, VI: Association for Supervision and Curriculum Development.

Ayers, W., Quinn, T. M. y Stovall, D. (2009). Handbook of social justice in education. Londres: Routledge.

Belavi, G. y Murillo, F. J. (2016). Educación, democracia y justicia social. Revista Internacional de Educación para la Justicia Social (RIEJS), 5(1), 13-34. doi:10.15336/riejs2016.5.1

Bell, B. y Cowie, B. (2001). The characteristics of formative assessment in science education. Science education, 85(5), 536-553. doi:10.1002/sce.1022

Benhabib, S. (2006). Las reivindicaciones de la cultura. Igualdad y diversidad en la era global. Buenos Aires: Katz.

Biesta, G. J. J. (2015a). Beyond learning. Democratic education for a human future. Boulder, CO: Paradigm Publishers.

Biesta, G. J. J. (2015b). Education, Measurement and the Professions: Reclaiming a space for democratic professionality in education. Educational Philosophy and Theory, Junio, 1-16. doi:10.1080/00131857.2015.1048665

Black, P. y Wiliam, D. (2009). Developing the theory of formative assessment. Educational Assessment, Evaluation and Accountability, 21(1), 5-31. doi:10.1007/s 1 1092-008-9068-5

Bolívar, A. (2005). Equidad educativa y teorías de la justicia. REICE: Revista Iberoamericana sobre Calidad, Eficacia y Cambio en Educación, 3(2), 42-69. 
Brisolera, S. A. (1998). The history of participatory evaluation and current debates in the field. En E. Whitmore (Ed.), Understanding and Practicing Participatory Evaluation. New Directions for Evaluation, $N^{o} 80$ (pp. 25-41). San Francisco, CA: Jossey-Bass.

Burke, B. (1998). Evaluating for change: Reflections on a participatory methodology. In E. Whitmore (Ed.) Understanding and Practicing Participatory Evaluation (pp. 43-56). San Francisco, CA: Jossey-Bass.

Cabrera, F. y Bordas, M. I. (2001). Estrategias de evaluación de los aprendizajes centradas en el proceso. Revista Española de Pedagogía, 59(218), 25-48.

Cousins, J. B. y Whitmore, E. (1998). Framing participatory evaluation. New Directions For Evaluation, 80, 5-23. doi:10.1002/ev.1114

Darling-Hammond, L. (1994). Performance-based assessment and educational equity. Harvard Educational Review, 64(1), 5-31.

Darling-Hammond, L. y Snyder, J. (2000). Authentic assessment of teaching in context. Teaching and Teacher Education, 16(5), 523-545. doi: 10.12691/education-3-12B-3

Dochy, F., Segers, M. y Dierick, S. (2002). Nuevas vías de aprendizaje y enseñanza y sus consecuencias: una nueva era de evaluación. Revista de Docencia Universitaria, 2(2), 13-29.

Dochy, F., Segers, M. y Sluijmans, D. (1999). The use of self-, peer- and co-assessment in higher education: a review. Studies in Higher Education, 24(3), 331-350. doi:10.1080/03075079912331379935

Duncan-Andrade, J. M. R. y Morrell, E. (2008). The art of critical pedagogy: possibilities for moving from theory to practice in urban schools. Berna: Peter Lang.

Everitt, A. (1996). Developing critical evaluation. Evaluation, 2(2), 173-188.

Everitt, A. y Hardiker, P. (1996). Evaluating for good practice. Basingstoke: Macmillan.

Fay, B. (1987). Critical social science: liberation and its limits. Cambridge: Polity.

Fraser, N. (2008). Scales of Justice: reimagining political space in a globalizing world. Cambridge: Polity.

Fraser, N. y Honneth, A. (2003). Redistribution or recognition? A political-philosophical exchange. Londres: Verso Press.

Freire, P. (1972). Teoría y práctica de la liberación. Madrid: Marsiega.

Freire, P. (1978). La educación como práctica de la libertad. Madrid: Siglo XXI editores.

Freire, P. (1979). Pedagogía del oprimido. Ciudad de México: Siglo XXI.

Friedrich, D., Jaastad, B. y Popkewitz, T. S. (2010). Democratic education: an (im)possibility that yet remains to come. Educational Philosophy and Theory, 42(5-6). doi:10.1111/j.14695812.2010.00686.x

Frierson, H.T., Hood, S. y Hughes, G. (2002). Strategies that address culturally responsive evaluation. Arlington, VA: National Science Foundation.

Gay, G. (2010). Culturally responsive teaching: Theory, research, and practice. Nueva York: Teachers College Press.

Giroux, H. A. (1983). Theory and resistance in education: a pedagogy for the opposition. South Hadley, MA: Bergin \& Garvey.

Giroux, H. A. (1988). Teachers as intellectuals: toward a critical pedagogy of learning. Westport, CO: Greenwood Publishing Group. 
Giroux, H. A. (2003). Pedagogía y política de la esperanza. Buenos Aires: Amorrortu.

Goodwin, A. L. (2012). Assessment for equity and inclusion: embracing all our children. Londres: Routledge.

Greene, J.C. (2006). Evaluation, democracy, and social change. En I. Shaw, J. Greene y M. Mark (Eds.), The Sage handbook of evaluation (pp. 118-140). Nueva York: Sage.

Griffin, P., McGaw, B. y Care, E. (2012). Assessment and teaching of 21 st century skills. Dordrecht: Springer.

Gulikers, J.T., Bastiaens, T.J. y Kirschner, P.A. (2004). A five-dimensional framework for authentic assessment. Educational Technology Research and Development, 52(3), 67-86. doi: $10.1007 / \mathrm{BFO} 2504676$

Gutmann, A. (1987). Democratic education. Princeton, NJ: Princeton University Press.

Gutmann, A. y Thompson, D. (2009). Why deliberative democracy? Princeton, NJ: Princeton University Press.

Hackman, H. W. (2005). Five essential components for social justice education. Equity $\mathbb{E}^{\circ}$ Excellence in Education, 38(2), 103-109. doi:10.1080/10665680590935034

Hargreaves, E. (2007). The validity of collaborative assessment for learning. Assessment in Education, Principles Policy and Practice, 14(2), 185-199. doi:10.1080/09695940701478594

Hodgen, J. (2007). Formative assessment. Tools for transforming school mathematics towards a dialogic practice. En D. Pitta-Pantazi y G. Philippou (Eds.), Proceedings of Fifth Congress of the European Society for Research in Mathematics Education (pp. 1886-1895). Larnaca: ERME.

Honneth, A. (1997). La lucha por el reconocimiento: por una gramática moral de los conflictos sociales. Barcelona: Editorial Crítica.

Honneth, A. (2007). Reificación. Un estudio en la teoría del reconocimiento. Madrid: Katz.

House, E. (1997). Evaluación, ética y poder. Madrid: Morata.

House, E. y Howe, K. (2000a). Deliberative democratic evaluation in practice. Education and Human Services, 49, 409-421. doi: 10.1007/0-306-47559-6_22

House, E. R. y Howe, K. R. (2000b). Deliberative democratic evaluation. En K. E. Ryan y L. DeStefano (Eds.), Evaluation as a democratic process: promoting inclusion, dialogue, and deliberation. (pp. 3-12). San Francisco, CA: Jossey-Bass.

Irvine, J. y Armento, B. (2001). Culturally responsive teaching: lesson planning for elementary and middle grades. Nueva York: McGraw-Hill.

Jackson, E. T. y Kassam, Y. (1998). Knowledge shared: participatory evaluation in development cooperation. Hartford, CO: IDRC.

Janesick, V. J. (2006). Authentic assessment primer. Berna: Peter Lang.

Janesick, V. J. (2008). Reflexiones sobre la violencia de las pruebas de evaluación estandarizada y la naturaleza tranquilizadora de la pedagogía crítica. En P. Mclaren y J.L. Kincheloe (Eds.), Pedagogía Crítica. De qué hablamos, dónde estamos (pp. 323- 337). Barcelona: Graó.

Kincheloe, J. L. (2008). Critical pedagogy primer. Berna: Peter Lang.

King M., Schroeder, J. y Chawszczewsky, D. (2001). Authentic Assessment and Student Performance in Inclusive Schools. Research Institute on Secondary Education Reform for Youth with Disabilities Brief, 5, 1-15. 
King, J. A., Cousins, J. B. y Whitmore, E. (2007). Making sense of participatory evaluation: Framing participatory evaluation. New Directions for Evaluation, 114, 83-105. doi: $10.1002 /$ ev.226

Knight, T. y Pearl, A. (2000). Democratic education and critical pedagogy. The Urban Review, 32(3), 197-226. doi:10.1023/A:1005177227794

Kozleski, E.B. (2010). Culturally responsive teaching matters. Tampe, AZ: Arizona State University

Leach, L., Neutze, G. y Zepke, N. (2001). Assessment and empowerment: some critical questions. Assessment and Evaluation in Higher Education, 26(4), $293-305$. doi:10.1080/02602930120063457

Lejk, M. y Wyvill, M. (2001). Peer assessment of contributions to a group Project: a comparison of holistic and category-based approaches. Assessment and Evaluation in Higher Education, 26(1) 61-72. doi:10.1080/02602930020022291

Lingard, B. y Mills, M. (2007). Pedagogies making a difference: issues of social justice and inclusion. International Journal of Inclusive Education, 11(3), 233-244. doi:10.1080/13603110701237472

Lingard, B., Mills, M. y Hayes, D. (2000). Teachers, school reform and social justice: challenging research and practice. The Australian Educational Researcher, 27(3), 101-115. doi:10.1007/BFo32 19733

Lupton, R. (2005). Social justice and school improvement: improving the quality of schooling in the poorest neighbourhoods. British Educational Research Journal, 31(5), 589-604. doi:10.1080/01411920500240759

McLaren, P. (1997). Critical pedagogy. Teaching Education, 9(1), 1-7.

McLaren, P. (2003). Critical pedagogy: a look at the major concepts. The Critical Pedagogy Reader, 1, 1-23.

McLaren, P. (2015). Life in schools: an introduction to critical pedagogy in the foundations of education. Londres: Routledge.

McLaren, P. y Kincheloe, J. L. (2007). Critical pedagogy: where are we now?. Berna: Peter Lang.

Mertens, D. M. (1999). Inclusive evaluation: implications of transformative theory for evaluation. American Journal of Evaluation, 20(1), 1-14.

Mertens, D. M. (2003). The inclusive view of evaluation: visions for the new millennium. En S. I. Donaldson y M. Scriven (Eds.), Evaluating social programs and problems: visions for the new millennium (pp. 91-107). Mahwah, NJ: Lawrence Erlbaum Associates.

Mezirow, J. (2000). Learning as transformation: critical perspectives on a theory in progress. San Francisco, CA: Jossey-Bass.

Moreno-Olivos, T. (2004). Evaluación cualitativa del aprendizaje: enfoques y tendencias. Revista de la Educación Superior, 33(3), 93-110.

Moreno-Olivos, T. (2010). Lo bueno, lo malo y lo feo: las muchas caras de la evaluación. Revista Iberoamericana de Educación Superior, 2(1), 84-97.

Mueller, J. (2006). The authentic assessment toolbox. Recuperado de http://jonathan.mueller.faculty.noctrl.edu/toolbox/standardtypes.htm

Murillo, F.J. (2004). Equidad en educación. REICE. Revista Electrónica Iberoamericana sobre Calidad, Eficacia y Cambio en Educación, 2(1), 1-24. 
Murillo, F. J. y Duk, C. (2012). Una evaluación inclusiva para une educación inclusiva. Revista Latinoamericana de Educación Inclusiva, 6(1), 11-13.

Murillo, F. J. y Hernández-Castilla, R. (2011). Hacia un concepto de justicia social. REICE. Revista Electrónica Iberoamericana sobre Calidad, Eficacia y Cambio en Educación, 9(4), 7-23.

Murillo, F. J. y Hernández-Castilla, R. (2014). Liderando escuelas justas para la justicia social. Revista Internacional de Educación para la Justicia Social (RIEJS), 3(2), 13-32.

Murillo, F. J. e Hidalgo, N. (2015a). Enfoques fundamentantes de la evaluación de estudiantes para la justicia social. Revista Iberoamericana de Evaluación Educativa, 8(1), 43-61.

Murillo, F. J. e Hidalgo, N. (2015b). Dime cómo evalúas y te diré qué sociedad construyes. Revista Iberoamericana de Evaluación Educativa, 8(1), 5-9.

Murillo, F.J. y Román, M. (2008). La evaluación educativa como derecho humano. Revista Iberoamericana de Evaluación Educativa, 1(1), 1-5.

Nicol, D. J. y Macfarlane-Dick, D. (2006). Formative assessment and self-regulated learning: a model and seven principles of good feedback practice. Studies in Higher Education, 31(2), 199-2 18. doi:10.1080/03075070600572090

North, C.E. (2006). More than words? delving into the substantive meaning(s) of "social justice" in education. Review of Educational Research, 76(4), 507-535. doi: $10.3102 / 00346543076004507$

Pfeiffer, M. L. (2007). En y por una educación con alto sentido social. Recuperado de http://goo.gl/NdsxDF

Popham, W. J. (2011). Transformative assessment in action: An inside look at applying the process. Alexandria, JE: ASCD.

Rawls, J.A. (1971). A theory of justice. Cambridge, MA: Harvard University Press.

Rawls, J.A. (2001). Justice as fairness: a restatement. Cambridge, MA: Harvard University Press.

Rennert-Ariev, P. (2005). A theoretical model for the authentic assessment of teaching. Practical Assessment Research E Evaluation, 1O(2), 151-163. doi:10.1207/s15324818ame1002_4

Ruitenberg, C. (2009). Educating political adversaries: Chantal Mouffe and radical democratic citizenship education. Studies in Philosophy and Education, 28(3), 269-281. doi:10.1007/s $11217-008-9122-2$

Ryan, K. E. (2004). Serving public interests in educational accountability: alternative approaches to democratic evaluation. American Journal of Evaluation, 25(4), 443-460. doi: $10.1177 / 109821400402500403$

Ryan, K., Greene, J., Lincoln, Y., Mathison, S. y Mertens, D. M. (1999). Advantages and challenges of using inclusive evaluation approaches in evaluation practice. The American Journal of Evaluation, 19(1), 101-122. doi:10.1177/109821409801900111

Sabirón, F. (1999). El discurso y la práctica en evaluación. Propuesta para la deconstrucción y reconstrucción de las prácticas evaluadoras. Zaragoza: ICE- Universidad de Zaragoza.

Sadler, D. R. (2009). Transforming holistic assessment and grading into a vehicle for complex learning. En G. Joughin (Ed.), Assessment, learning and judgement in higher education (pp. 119). Dordrecht: Springer.

Santiuste, V. y Arranz, M. L. (2009). Nuevas perspectivas en el concepto de evaluación. Revista de Educación, 350, 463-476. 
Santos Guerra, M. A. (1993). La evaluación: un proceso de diálogo, comprensión y mejora. Investigación en la Escuela, 20, 23-38.

Santos Guerra, M. A. (2003). Una flecha en la diana: La evaluación como aprendizaje. Madrid: Narcea.

Saunders, M. (2006). The 'presence' of evaluation theory and practice in educational and social development: Toward an inclusive approach. London Review of Education, 4, 197-215.

Schniedewind, N. y Davidson, E. (2006). Open minds to equality: a sourcebook of learning activities to affirm diversity and promote equity. Milwaukee, WI: Rethinking Schools.

Shepard, L. A. (2005). Linking formative assessment to scaffolding. Educational Leadership, 63(3), 66-70.

Shepard, L. A. (2000). The role of classroom assessment in teaching and learning. Nueva York: Teachers College Press.

Sleeter, C. (2013). Teaching for social justice in multicultural classrooms. Multicultural Education Review, 5(2), 1-19. doi:10.14328/MER.2013.09.30.01

Smyth, J. (2012). The socially just school and critical pedagogies in communities put at a disadvantage. Critical Studies in Education, 53(1) 9-18. doi:10.1080/17508487.2012.635671

Stern, J. y Backhouse, A. (2011). Dialogic feedback for children and teachers: evaluating the 'spirit of assessment'. International Journal of Children's Spirituality, 16(4), 331-346. doi:10.1080/1364436X.2011.642853

Stowell, M. (2004). Equity, justice and standards: assessment decision making in higher education. Assessment and Evaluation in Higher Education, 29(4), 495-510. doi:10.1080/02602930310001689055

Straume, I. (2015) Democracy, education and the need for politics. Studies in Philosophy and Education, 35(1), 29-45. doi: 10.1007/s11217-015-9465-4

Swaffield, S. (2011). Getting to the heart of authentic assessment for learning. Assessment in Education: Principles, Policy \& Practice, 18(4), 433-449. doi: 10.1080/0969594X.2011.582838

Swan, K., Shen, J. y Hiltz, S. R. (2006). Assessment and collaboration in online learning. Journal of Asynchronous Learning Networks, 1O(1), 45-62. doi:10.1080/14748460600855526

Taylor, C. (2003). El multiculturalismo y "la política del reconocimiento". Ciudad de México: Fondo de Cultura Económica.

Thomas, V.G. (2004). Building a contextually responsive evaluation framework: Lessons from working with urban school interventions. En V.G. Thomas y F.I. Stevens (Eds.), Coconstructing a contextually responsive evaluation framework (pp. 3-23). San Francisco, CA: Jossey-Bass.

Thomas, V. G. y Stevens, F. I. (2004). Coconstructing a contextually responsive evaluation framework. San Francisco, CA: Jossey-Bass.

Torrance, H. (1995). Evaluating authentic assessment. Buckingham: Open University Press.

Torrance, H. (2012). Formative assessment at the crossroads: conformative, deformative and transformative assessment. Oxford Review of Education, 38(3), 323-342. doi: $10.1080 / 03054985.2012 .689693$

Vu, T. T. y Dall'Alba, G. (2014). Authentic assessment for student learning: an ontological conceptualisation. Educational Philosophy and Theory, 46(7), 778-791. doi:10.1080/00131857.2013.795110 
Waters, G. A. (1998). Critical evaluation for education reform. Education Policy Analysis Archives, 6, 20. doi:10.14507/epaa.v6n20.1998

Wehlburg, C. (2008). Promoting integrated and transformative assessment: a deeper focus on student learning. San Francisco, CA: Jossey-Bass.

Whitmore, E. (ed.). (1998). Understanding and practicing participatory evaluation. San Francisco, CA: Jossey-Bass.

Wiggins, G. (1989). A true test: toward more authentic and equitable assessment. Phi Delta Kappan, 70, 703-713. doi:10.1177/003172171109200721

William, D. (2007). Assessment for learning: why, what and how. Londres: Institute of Education.

Wink, J. (2005). Critical pedagogy: notes from the real world. Boston: Pearson.

Young, I. M. (2000). La justicia y la política de la diferencia. Madrid: Ediciones Cátedra.

Young, I. M. (2011). Responsabilidad por la justicia. Madrid: Paidós.

Zajda, J., Majhanovich, S. y Rust, V. (2006). Education and social justice. Dordrecht: Springer.

\section{Breve CV de los autores}

\section{Nina Hidalgo Farran}

Doctorando en Educación en el área de Métodos de Investigación y Diagnóstico en Educación en la Universidad Autónoma de Madrid, con una beca de doctorado de carácter competitivo otorgada por el Ministerio. Es diplomada en Ciencias de la Educación en la especialidad de Maestra de Educación Infantil por la Universidad de Lleida y licenciada en Psicopedagogía por la Universidad Autónoma de Madrid. Es miembro del grupo de investigación Cambio Educativo para la Justicia Social (GICE) y miembro del equipo editorial de la Revista Internacional de Educación para la Justicia Social. Asimismo es editora de la Revista de la Revista Iberoamericana de Evaluación Educativa. Cuenta con diversas publicaciones, como artículos, recensiones y capítulos de libro. Página personal: http://www.gice-uam.es/Nina.Hidalgo. ORCID ID: 0000-0002o056-8566. Email: nina.hidalgo@uam.es.

\section{F. Javier Murillo Torrecilla}

Profesor titular del Área de Métodos de Investigación y Diagnóstico en Educación de la Universidad Autónoma de Madrid. Coordinador del Doctorado en Educación de la UAM. Coordinador del grupo de Investigación Cambio Educativo para la Justicia Social (GICE).

Fue Coordinador General del Laboratorio Latinoamericano de Evaluación de la Calidad de la Educación (LLECE), de la UNESCO, y Director de Estudios del Centro de Investigación y Documentación Educativa (CIDE) del Ministerio de Educación de España. Es Coordinador de la Red Iberoamericana de Investigación sobre Cambio y Eficacia Escolar (RINACE), Director de REICE. Revista Iberoamericana sobre Calidad, Eficacia y Cambio en Educación, de la Revista Iberoamericana de Evaluación Educativa y de la Revista Internacional de Educación para la Justicia Social. Página de docencia: http://www.uam.es/javier.murillo. ORCID ID: 0000-0002-8003-4133. Email: javier.murillo@uam.es. 